\title{
Adaptive and Self Healing Routing for Mobile Ad Hoc Networks Using Cross Layer Design
}

\author{
S. Menaka ${ }^{1}$ and M. K. Jayanthi ${ }^{2}$ \\ ${ }^{1}$ School of Information Technology and Engineering, VIT University, Vellore, Tamil Nadu, India \\ ${ }^{2}$ Department of Computer Science and Engineering, King Khalid University, Abha, Saudi Arabia
}

\begin{abstract}
The construction of reliable and stable routes in a mobile ad hoc network is one of the primary research issues in equipping each device to continuously maintain the information required to properly route traffic. Mobility of nodes often leads to link failures and hence requires route reconstruction to resume the communication between the nodes. The stability factor of a route can reduce the number of times the route is changed or reconstructed. This paper presents a novel idea for discovering a stable set of routes using the metrics from multiple layers rather than depending on network layer along with a finite set of parameters to qualify a link or connecting to a node. The link stability factor and link received signal strength are considered as the main metrics to qualify the stability of a route, derived from the physical and data link layer based on bit or packet error rate, retrieved from the soft output decoder. The simulation results based on the analysis of the proposed algorithm prove to be more efficient in terms of discovering stable routes, reducing frequent reconstruction of routes and hence improving the overall performance of the network.
\end{abstract}

Keywords: mobility, link stability, cross layer metrics, received signal strength

\section{Introduction}

The recent enhancements of wireless communication networks is the transmission of realtime information, including audio and video data which are categorized as delay sensitive communication. Hence the stability of the communication channel becomes a mandatory requirement for identifying a stable route in a multihop mobile ad hoc network. An ad hoc network is formed by communication of mobile terminals directly, without the intervention of a central unit where every node in the network performs the routing function to identify the route to the corresponding destination node to exchange data. The mobile node in an ad hoc network causes variation in the quality of wireless link signals because of the changes in the propagation path loss, shadowing effect, multipath fading and interference. All these results in the breakage of the link in a multihop route, leading to router failure resulting in the disruption of the communication, incurring delay until the connectivity is resumed. Therefore, stability of the route is a challenging issue in routing. As pointed by Bahador Amiri et al. (2011), it contributes to the majority of the overall performance of the network. In mobile ad hoc networks, there may exist multiple paths to a destination from the source, selecting the best route in terms of reliability of the links, which was observed to be a crucial task for any routing protocol. This process of identifying available paths and selecting a reliable and stable path is the goal of any routing algorithm. To achieve the objective of identifying the available route and selecting a stable route becomes a mandatory requirement in an ad hoc environment with high mobility. In a high mobility network, the adaptive routing plays a major role to sustain the communication, as commented by Hadi Sargolzaey et al. (2012). Hence, in this paper, we propose a routing scheme using multifactor metric to discover a stable route and an effective route maintenance phase to gracefully switch over to an alternate route, even before the connectivity gets disconnected due to the mobility of the nodes. 
MANET routing protocols are categorized into two classes, proactive and reactive routing. As given by the author Lorenzo Mucchi et al. (2012), proactive routing protocols have high control overhead due to maintenance of routes periodic probing of the route status, even when not required, while the reactive routing protocol incurs higher end to end delay due to the reformation of routes during communication. Hybrid routing protocols overcome the above drawbacks of proactive and reactive routing using the link state information to control the disconnectivity caused by node mobility and low power provided by the cross layer architecture, as investigated by Edward Y. Hua et al., (2008). Most widely used reactive routing protocols are AODV (Ad hoc On Demand Distance Vector) and DSR (Dynamic Source Routing) that identifies multipath routes stored in route cache as backup routes to utilize as alternate routes during route failures discussed by Mehajabeen Fatima et al. (2012). The above protocols use the number of hops required to reach the destination as the routing metric acquired from the network layer which doesn't include all the other features of mobile terminals operating in a high mobility environment proposed by Lorenzo Mucchi et al., (2012). The above routing protocols prefer routes with minimum delay, without focusing on the stability of the routes caused by the mobility of the nodes. When a highly stable and reliable route is discovered, then the number of link failures and reconstruction of routes can be reduced. To reduce the frequent link breakage, it is required to design an efficient routing scheme to discover a reliable and stable route. This paper proposes a technique based on the cross layer design to discover stable routes using metrics from multiple layers. The algorithm is not implemented from the scratch, rather it is analyzed as a modified version of the Dynamic Source Routing protocol.

Further sections of the paper is organized as Section 2 which discusses the literature on routing using cross layered architecture, Section 3 presents the details on the proposed cross layer metric to discover stable routes and Section 4 discusses the simulation results of the proposed algorithm compared with AOMDV and DSR, with Section 5 giving the concluding remarks.

\section{Cross Layer Routing}

Cross Layer design is mainly used to acquire the node's mobility information in order to enhance the performance of the reactive routing protocol, to switch over an alternate route when there is a warning of disruption of active route, to avoid loss of data packets when the link fails. A number of cross layer metrics are proposed by various researchers, which is discussed in this section. The principle of cross layer design is to make routing decisions based on multiple values obtained from different layers, instead of metric used only from the routing layer according to discussion by Mehajabeen Fatima et al. (2012). Figure 1 illustrates the scheme of information exchange among the multiple layers in a network stack.

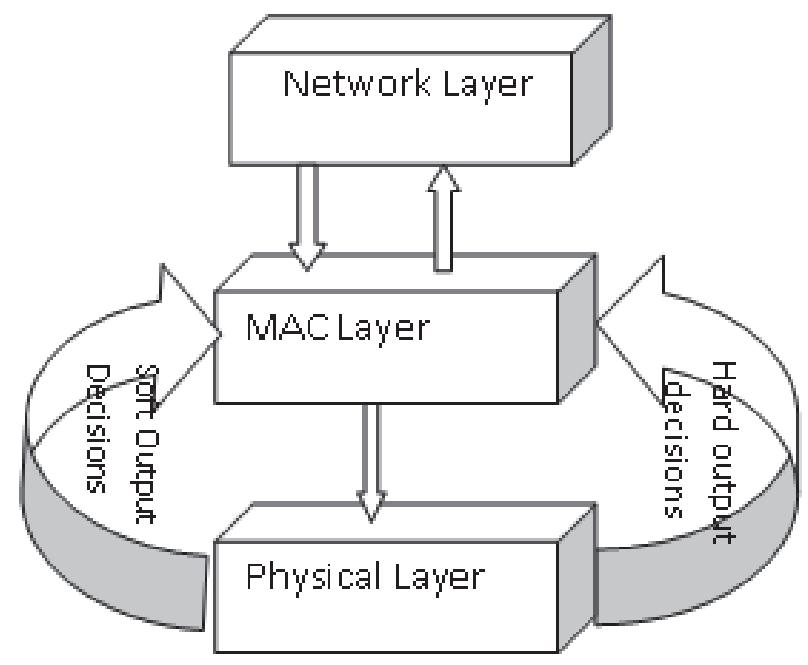

Figure 1. Illustration of interlayer communications in cross layer design.

A detailed review of reliability based routing protocols and their metrics is given in Hadi Sargolzaey et al. (2012). On the whole, the reliability metrics used are:

i) LET-Link Expiration Time from the network layer

ii) PLRT-Probabilistic Link Reliable Time from the network layer.

iii)LPER-Link Packet Error Rate from data link layer.

iv) RSS-Received signal strength from the physical layer.

In literature, all the aforementioned metrics enhance the reliability of the route and hence the 
performance of the network, but with varying levels of efficiency.

LET is a position based metric obtained at the network layer, assuming a free space propagation and the motion parameters of two neighboring nodes. This metric requires every node in MANET to be equipped with Global Positioning System (GPS) to retrieve the necessary parameters. In the article by Edward Y. Hua et al. (2008) LET is predicted as Link Life Time (LLT) as given by Merlinda Drini et al., (2009) and Hang Shen et al. (2008) from the mobility pattern, its velocity and hence the location of the node and remaining power information without the need for a GPS facility in the node.

PLRT is computed by averaging the measured link life time, by running an unreliable base protocol and deriving the link failure rate to compute the link reliability, as given by the system reliability equation as:

Probability (link_stability duration) $=e^{-\rho t}$, where $\rho$ (Rho) is the inverse of the lifetime of the link.

LPER is a measure of link reliability computed from the number of damaged receiving packets obtained from the data link layer ruled as: higher the value of the packet error rate, lower the reliability of the link that is transferred to the network layer to make the routing decision to select the link to include in the route which is a cross layer approach. Awadhesh Kumar et al. (2011) proposed the (LLTp) predicted link lifetime metric by considering the node's remaining power. The link failure due to node's mobility has not been focused in their work.

Investigations by B. Ramachandra et al. (2008) showed that the link RSS is acquired from the physical layer and transferred to the network layer. This implies that if the received signal strength of the link is below a threshold value, the link is rejected to route the data packet which is a cross layer approach. The RSS decreases as the node moves away from the transmission range, warning that the link associated could break the route and hence it is considered as one of the best metrics to select as an alternate route to sustain the data transmission.

Many protocols that utilize the information from multiple layers to make routing decisions have been proposed in literature like ABR - Associativity based routing and System Stability
Based Adaptive routing (SSA) as proposed by Husnain Mansoor et al. (2007). Improvements to the above routing protocols are contributed by proposing various other parameters like Expected Transmission count (ETX), expected transmission time (ETT) and weighted cumulative ETT (WCETT). The Metric of interference and channel switching (MIC) presented by Mohamed Asiri Bin et al. (2012) and Lian Qin et al., (2004) enhanced the performance in fixed ad hoc networks without any evidence of their performance in MANETs. Most of the proposed protocols use the parameters such as distance between nodes, mobility rates and energy consumption. Authors Hadi Sargolzaey et al., (2012) and Mohamed Asri Bin et al., (2012) presented the DXFP routing protocol adopting a cross layer metric conventional packet error rate, providing a robust method for path discovery and backup routes for continuity of the connectivity. This protocol takes only a single quantity of link quality at varying time, incurring higher signaling load along with the deterioration of the link due the powerful energy of the node. Edward Y. Hua et al., (2008) has given a proposal of cross layer self routing design by modifying the AODV protocol by using cross layer metric. They contributed towards the route maintenance of AODV to reduce frequent link breaks by computing the Link Life Time metric which was proposed by Geetha Nair, et al., (2012). Link Life Time metric is measured from the relative mobility, speed of the nodes and power management of transmission power control algorithms. All the above algorithms compute the stability factor of the link from the SNR of the data packet.

Author Kambiz Homayounfar, (2009) presented CLMRA algorithm, a cross layer route selection based on radio link stability; using two path metrics i.e., link instability index and path instability index for selecting a route. This technique incurs higher route acquisition latency and also identifies the single best route to the destination, which are the drawbacks of the CLMRA algorithm.

Chao Gu et al., (2013) proposed the duration of interference imposed by nodes as a cross layer metric to predict the link breakage frequency to discover the route. Their work involves setting up of threshold value to make routing decisions, 
which cannot be fixed as generalized value for all type of MANETs.

The authors Hang Shen et al., (2008) proposed the cross layer based metric "sfd", signal fading degree computed from the energy values of the node focus on the link breakage due to energy drain and did not much consider mobility of the nodes as a contributing factor for route failures.

From the above literature study it is evident that the node mobility induces varying links in terms of bandwidth and interference, which is a challenging aspect in MANET routing. This article presents a cross layer routing metric for route discovery and maintenance for MANETs.

\section{System Design}

The cross layer based reliable routing algorithm is designed using the metric from the physical layer, data link layer, and transferring it to the network layer to make the routing decisions. This algorithm involves the mobile node cooperatively to establish the route and introduces the radio link stability with extensive cross layer cooperation to establish reliable and stable routes. The present study is aimed at developing an algorithm by means of modifying the underlying principle of dynamic source routing protocol. As defined by Hang Shen et al., (2008), dynamic source routing is known for its benefits of routing in multiple paths, avoiding the overhead of new route discovery whenever the route in use breaks. The route discovery and route maintenance easily support unidirectional links and asymmetric routes. DSR is also compatible with inter-network routing consisting of different types of network. The only drawback of DSR could be reported as its performance is not par with other reactive routing protocols in a high mobility environment. Another reason for selecting DSR as base protocol is the considerable research being carried out on cross layer design protocol which is implemented with AODV, and limited work done with DSR.

The cross layer-dynamic source routing (CLDSR) has had two phases: Route Discovery and Route Maintenance. Figure 2 represents the architecture of the proposed algorithm.

The cross layer metrics discussed in the literature focus on the link lifetime based on received

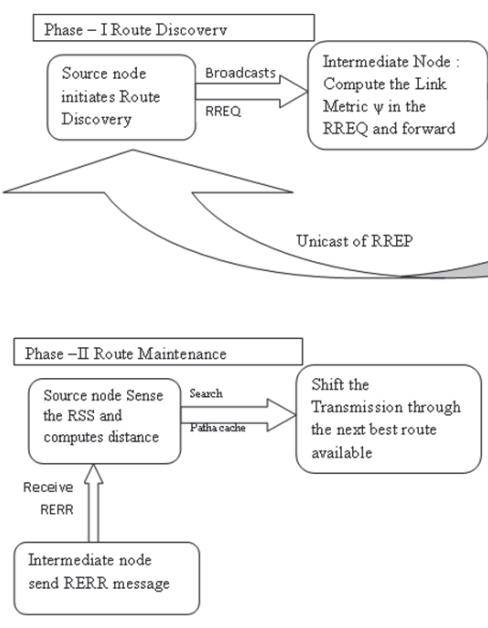

Figure 2. Process Diagram of CL-DSR.

signal strength, distance between the nodes, the number of damaged received packets which are contributed by SNR (Signal to noise ratio). In reality, fading effects due to mobility and multipath propagation also affect the transmission and cause fluctuation of signal strength leading to breakage of an active link, according to Justus Ch. Fricke, et al., (2007) and Eric Setton et al., (2005). Forward Error Correction (FEC) channel coding is used in digital transmission system for error correcting. It provides reliable information that can be retrieved from the soft outputs of the channel decoder. Figure 3 represents the transfer of required data to compute the metric to establish the route. In this approach of cross layer design, along with link SNR, the fading effect and FEC role are also considered in finding stable routes.

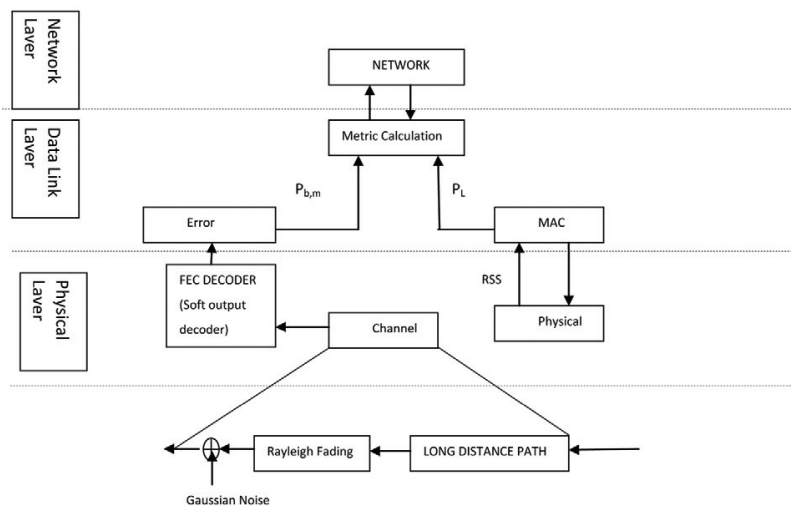

Figure 3. Data acquisition in a cross layer architecture. 


\subsection{Route Discovery in CL-DSR}

Route discovery consists of link metric computations to decide on the stability and quality of the link to prioritize the route. The components of the link metric are the link instability index contributed by the average bit error probability from the soft output decoder and path loss from the received signal strength.

Similar to DSR, the route discovery is initiated by the source only when it has to send data packets to the destination. The source node broadcasts a RREQ packet to its neighboring nodes. The receiving nodes of the RREQ packet will update the RREQ packet with its ID, next hop and link metric computed based on the CL-DSR rules. At the destination the node populates its RREP packet with both metric and unicasts to the source node.

The path instability index denoted by ' $\Psi$ ' indicates how instable the link is. Hence lower the value of $\Psi$ better the path. The source node on receiving the RREP packet from multiple routes selects the route with lowest path instability index and initiates the data transmission. The other routes are stored in the path cache in the ascending order of the path instability index.

\subsubsection{Computation of Link Metric}

The individual link metric is computed from the soft outputs provided by the channel decoder. The soft outputs are retrieved for the previous processing like equalization, detection and decoding and hence the impact of SNR, interference and fading are also included.

The source node is termed as $N_{s}$ while the destination node is denoted as $N_{d}$ and the intermediate node is identified as $N_{i}$. CL-DSR proposes the link metric as the link instability index denoted by ' $\psi$ ' as the cumulative value of the average bit error rate and their path loss is computed from received signal strength. The computation of average bit error rate is given by Justus Ch. Fricke, et. al, (2007). A multihop route consists of ' $n$ ' number of nodes connected by ' $n-1$ ' number of links. The link instability index of link ' $m$ ' between the intermediate node $N_{i-1}$ and $N_{i}$ is represented as

$$
\psi_{N i-1, N i}=P_{b, m}+P_{L}
$$

where $P_{b, m}$ is the average bit error probability of link ' $m$ ' and $P_{L}$ is the path loss incurred when receiving the RREQ at the node ' $i$ ' from the source node. The following sections demonstrate the computation of the link instability index components.

\section{A. Average Bit Error Probability}

The bit error probability (BEP) of $k^{\text {th }}$ information bit is computed from the log likelihood ratio of a given bit as

$$
P_{b, k}=\frac{1}{1+e^{\left|\cup_{k}\right|}}
$$

Averaging the value of equation (1) the overall $\mathrm{BEP}$ of a $\mathrm{K}$ bit word is obtained, from which average BEP of the transmission of the link $m$ is calculated as per equation (2).

$$
P_{b, m}=\frac{1}{K} \sum_{k=1}^{K} \frac{1}{1+e^{\left|\cup_{k}\right|}}
$$

\section{B. Computation of Path Loss}

When the source node emits the RREQ packet, it inserts its transmission power $\left(P_{T}\right)$ and the receiving node computes the path loss $\left(P_{L}\right)$ as the difference between the transmission power of the sender's node and its received signal strength $\left(P_{R}\right)$ obtained from the physical layer.

Path Loss is computed as

$$
P_{L}=P_{T}-P_{R}
$$

From equations (2) and (3) the link instability index $\psi$ is calculated as given in equation (4)

$$
\psi=P_{b, m}+P_{L}
$$

And henceforth the path instability index $\Psi$ is computed as

$$
\Psi=\sum_{i=1}^{n} \psi_{i}
$$

where $\mathrm{n}$ is the number of intermediate nodes along the route 


\subsection{Route Maintenance in CL-DSR}

This is the heart of any reactive routing protocol that reacts to the topology changes to maintain the connectivity among nodes. Route maintenance is the process of monitoring the route status and resuming the communication by selecting an alternate route from the path cache or reconstructing a new route. The link life time prediction scheme was earlier proposed by Edward Y. Hua et al. (2009) by using UKF prediction algorithm which has a drawback of periodic measurements involving resources. In this approach the route maintenance proposes a preemptive scheme of DSR, such that when the danger of link breakage is sensed in the current route, CL-DSR switches to the alternate route from the route cache, even before the connectivity is disrupted. According to CL-DSR, route maintenance also uses the cross layer metric to predict the link breakage and when there is a danger of link breakage it provides a graceful switch over of transmission from active route to the next best route as an alternate route from the route cache, even before the route gets disconnected. This method of preempting from the active route to alternate route the end to end delay due to link breakage is much reduced. The route, including the broken link is removed once the RERR message reaches the source node, as well as the other nodes using the link and hence reducing the presence of stale routes in the route cache, as proposed in our previous work. S. Menaka, et al., (2013). The link breakage is predicted from the mobility, speed of the node.

\subsubsection{Prediction of Link Breakages}

The mobility, speed of the node is computed by calculating the difference in distance between the nodes within a time interval $\partial$ and comparing it with transmission range $(R)$, to predict when the node moves away from the transmission range and thereby deriving the velocity of the mobility of the node. The distance between the nodes is computed as the ratio of transmitted power and received power, as given in equation (5). The received signal strength is obtained from the received MAC frames. Then the difference in the distance value in the time interval $(t, t+\partial t)$ is computed and compared with the transmission range $R$ to predict the link breakage. Figure 4 illustrates the node's mobility in the network

$$
d=P_{t} / P_{r}
$$

where ' $d$ ' is the distance between the nodes, $P_{t}$ and $P_{r}$ are the transmitted and received power, respectively

$d 1 \rightarrow$ distance at time ' $t$ '

$d 2 \rightarrow$ distance at time $t+\partial t$

If $(d 2-d 1)>R$, initiate the switch to another route

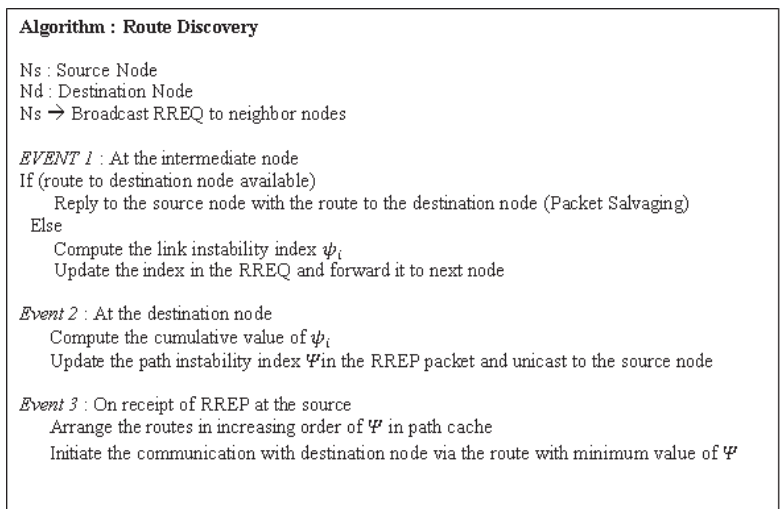

Figure 4. Route discovery in CL-DSR.

In Figures 5(a) and 5(b), when there is path from $\mathrm{S}$ to $\mathrm{D}$ via $\mathrm{A}-\mathrm{B}$, as the node ' $\mathrm{B}$ ' moves away from the transmission range of ' $A$ ' \& ' $D$ ' as in scenario 2 or if it is on low battery, the received signal strength diminishes. This effect traverses to the source node, by which the source node gracefully switches over from the current route to the next best route available from the path cache. Meanwhile, the intermediate node 'A' estimates the distance between A and $\mathrm{B}$ from equation 5 . If the difference in the distance to the transmission range is relatively high, then the node ' $\mathrm{A}$ ' senses the link breakage

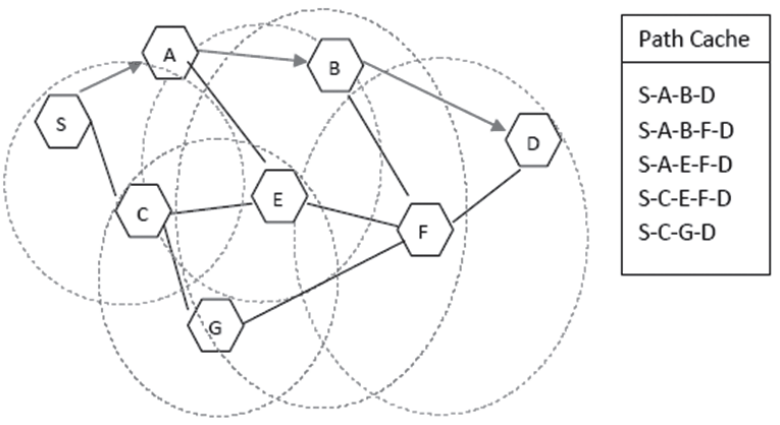

Figure 5(a). Route maintenance - Scenario 1. 


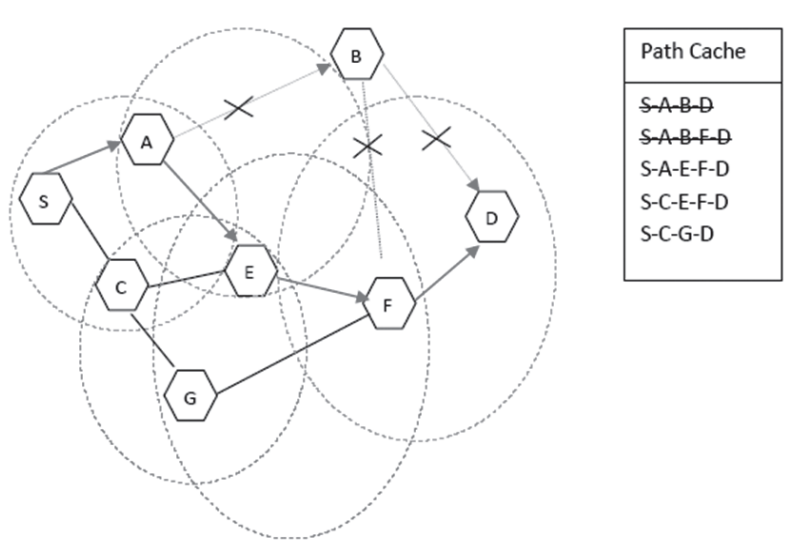

Figure 5(b). Route maintenance in CL-DSR.

and shifts its transmission via nodes ' $\mathrm{E} \& \mathrm{~F}$ ' from its path cache, to transmit the data packets in its buffer. After switching to the route via ' $E$ ' $\&$ 'F', node 'A' informs the source of link breakage of (A-B) with the RERR message based on which route is removed from the route cache.

This methodology of route maintenance can warn the nodes of danger of link breakage, either due to the mobility or due to the drain of battery power.

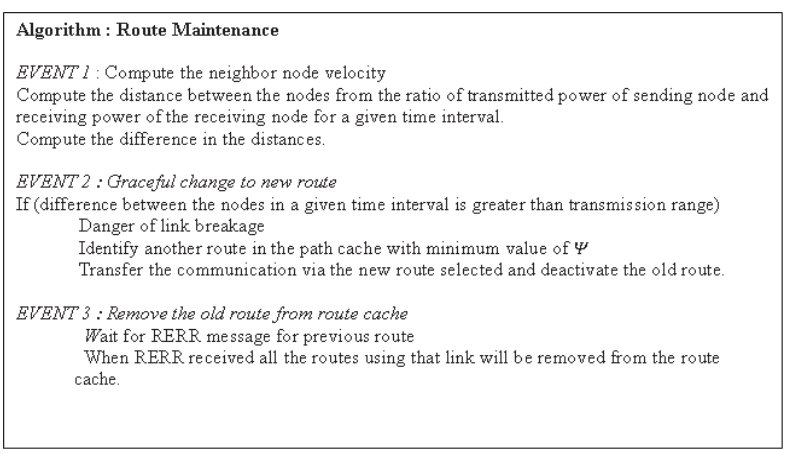

Figure 6. Route maintenance algorithm in CL-DSR.

\section{Simulation}

Simulation of the proposed routing scheme is implemented and evaluated in NS3. The algorithm is studied with 100 nodes in an area of 1500 square meters with the bandwidth of $2 \mathrm{MHz}$ shared media in a two ray propagation model. Random way point mobility model is used for the mobility pattern in a constant bit rate traffic of generating 4 packets per second of 512 bytes size. A simulation is run for 1000 seconds with a constant pause time of 4 seconds. Table 1 gives the summary of simulation setup parameters. Each simulation is run for 10 iterations to increase the accuracy of the values.

\begin{tabular}{|l|l|}
\hline Parameters & Values \\
\hline \hline No: of Nodes & 100 \\
\hline Topology area & 1500 x 1500 \\
\hline MAC & 802.11 \\
\hline Radio range & $250 \mathrm{~m}$ \\
\hline Simulation time & 1000 secs \\
\hline Traffic Source & CBR \\
\hline Packet Size & 512 bytes \\
\hline Packet Rate & 5 pkts/s \\
\hline Mobility Model & Random Way Point \\
\hline Propagation Model & Two Ray Ground \\
\hline
\end{tabular}

Table 1. Simulation environment.

The performance evaluation is analyzed comparing the proposed technique with AOMDV and DSR which are multipath routing algorithms using the following parameters

i) Link failure frequency measured for varying speeds of the mobility of the nodes

ii) Routing overhead measured from the number of control packets exchanged

iii) End to end delay

Link failure frequency: The mobility, speed of the nodes varies between 0-25 meters per second and as the node moves away from the transmission range, the link breaks, resulting in route failure. The number of such link breakages characterizes the stability of the route.

Routing overhead: When there is a link breakage, the route is disconnected and an alternate route is selected, involving exchange of routing control messages known as routing overhead that is computed in terms of data bits.

End to end delay: the ratio between time difference between every CBR packet sent and received and the total time difference over the total number of CBR packets received. It refers to the time taken for a packet to be transmitted across a network from source to destination and is given as:

$D_{\text {etoe }}=N\left(D_{\text {trans }}+D_{\text {prop }}+D_{(\text {proc })}\right.$

where

$D_{\text {trans }}=$ Transmission delay

$D_{\text {prop }}=$ Propagation delay 
$D_{\text {proc }}=$ Processing delay

A snapshot of the simulation run is given in Figure 7.

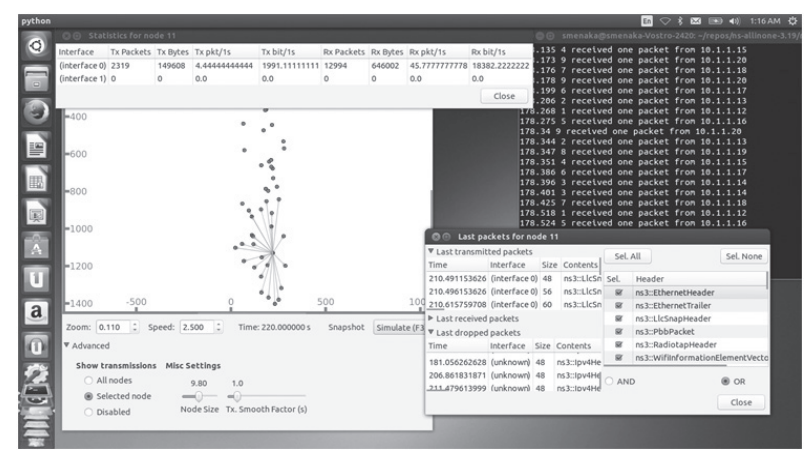

Figure 7. Snapshot of simulation run in NS3.

The following section discusses the results obtained from the simulation.

\subsection{Results and Discussion}

The results of the simulation of the proposed algorithm compared with AOMDV and DSR are shown in Figures 8 to 10 . Results exhibited a considerable improvement in the performance of the proposed algorithm compared to the traditional AOMDV and DSR.

It can also be noted in Figure 8 that the average link failure frequency derived from the number of routes used for the simulation period. The more routes are used, the more link failures occur. From the graphical representation given in Figure 8 it is evident that CL-DSR outperforms the other two protocols in terms of stability of the routes selected initially. Since the route is more stable in CL-DSR, the number of routes used is $16 \%$ and $28 \%$ lesser than DSR and AOMDV respectively, indicating that the link failure frequency is considerably less.

Frequent changes in the routes incur the exchange of control data which adds to the routing overhead. Figure 9 shows that $30 \%$ of routing overhead is reduced in case of CL-DSR, as the need for reconstruction of routes is minimized because of selecting a stable route instead of a short route, based on the number of hops.

From Figure 10 it is understood that the most essential requirement of communication, the end to end delay, is very much reduced as a result

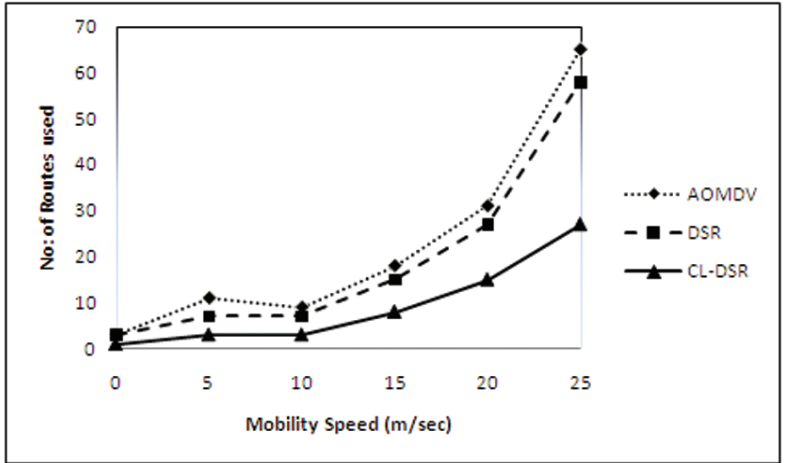

Figure 8.

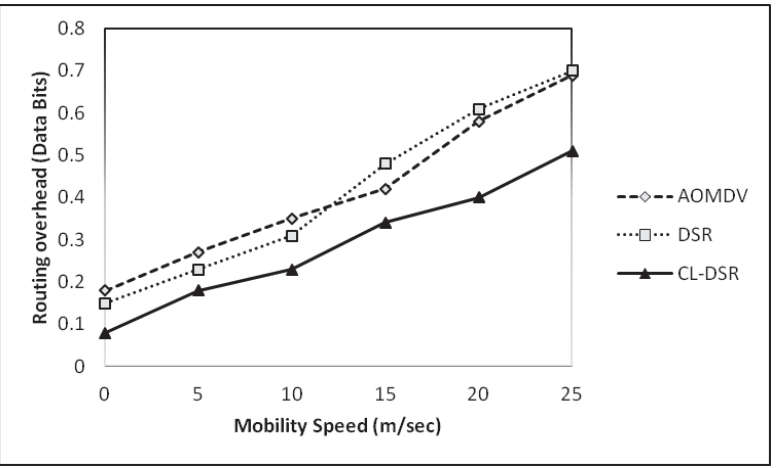

Figure 9.

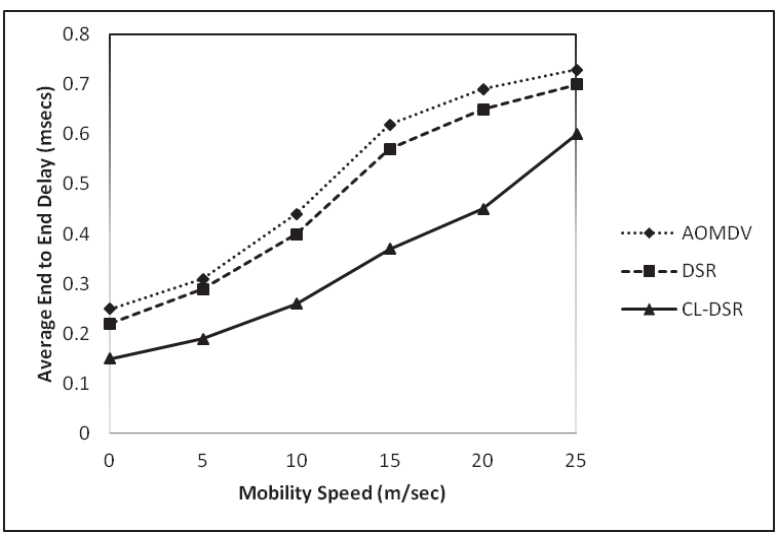

Figure 10.

of the impact of the above two factors. CLDSR incurs 30-35\% lesser delay than the other two protocols. Higher stability of routes and lesser end to end delay in the case of CL-DSR outperform DSR \& AOMDV routing protocols

The above results are much more evident to prove the proposed metrics and methodology to discover a more stable and reliable route which improves the performance of the routing protocol. 


\section{Conclusions and Future Work}

It can be concluded from the study that a cross layer, multi metric routing scheme for mobile ad hoc networks appeared more suitable for the high mobility environment. This approach helps to discover routes using cross layer design and multiple metrics to qualify the routes. This guarantees stable, reliable routes and an efficient route repair in case of link breakage due to the mobility of the nodes. The proposed technique of discovering stable routes by the source on demand exhibits a considerable improvement on the performance factors with reduced route traffic, route failure frequency and end to end delay as compared to other commonly used source routing protocols such as AOMDV and DSR. This methodology of route discovery may incur delay to initiate the communication, but does not degrade the overall performance of the routing protocol and hence the network performance. This initial delay is due to metric computations involved in route discovery and, at the same time, it identifies a stable and reliable route. In this work the pause time is considered constant, and hence further study is motivated to test the same methodology with the varying pause time and its impact on the network performance.

\section{References}

[1] A. KUMAR, DR. N. TyAGI, Cross-layer self organized routing protocol for MANET. Proc. of CSIT, International Conference on Computer Communication and Management, 5, IACSIT Press, Singapore 2011.

[2] B. RAMACHANDRAn, S. SHANMUGAVEL, Received Signal Strength Based Cross-Layer Designs for Mobile Ad Hoc Networks. IETE Technical Review, $\mathbf{5}(4), 2008$

[3] B. AMIRI, H. R. SADJADPOUR1, J. J. GARCIA-LUNAACEVES, Cross-layer design of outage optimum routing metric for wireless ad hoc networks. Wireless Communications And Mobile Computing, 2011.

[4] C. GU, Q. ZHU, A Cross-Layer Routing Protocol for Mobile Ad Hoc Networks Based on Minimum Interference Duration. Proceedings of the 2nd International Conference on Computer Science and Electronics Engineering (ICCSEE 2013).

[5] E. Y. HuA, Z. J. HAAS, Fellow, Cross-Layer Self Routing: a self-managed routing approach for MANETs, Networking and Communications. International Conference on Wireless and Mobile Computing, WIMOB '08. IEEE 2008.
[6] E. Y. HuA, Z. J. HAAS, An Algorithm for Prediction of Link Lifetime in MANET Based on Unscented Kalman Filter. IEEE COMMUNICATIONS LETTERS, 13(10), OCTOBER 2009.

[7] E. Setton, T. Yoo, X. Zhu, A. Goldsmith, B. GIROD, Cross-Layer Design of Ad Hoc Networks for Real-Time Video Streaming. IEEE Wireless Communications, August 2005.

[8] G. NAIR, DR. N. J. R. MuniRAJ, Prediction Based Link Stability Scheme for Mobile Ad Hoc Networks. IJCSI International Journal of Computer Science Issues, Vol. 9, Issue 6, No 3, November 2012.

[9] H. Sargolzaey, B. Mohd Ali, S. Khatun, A Cross Layer Metric for Discovering Reliable Routes in Mobile Ad Hoc Networks. Wireless Pers Commun, 66, 207-216, 2012.

[10] H. Shen, G. BAI, J. WANG, Y. Jin, J. TAO, Performance Study of a Cross-Layer Based Multipath Routing Protocol for IEEE 802.11e Mobile Ad Hoc Networks. International Journal on Communications, Network and System Sciences, 4, 285-385, 2008.

[11] H. Mansoor Ali, Amina Meraihi Naimi, AnTHONY Busson, VERONIQUE VEQUE, Signal Strength Based Link Sensing for Mobile Ad-Hoc Networks, 2007.

[12] J. Ch. Fricke, M. I. RAFIQUe, P. A. HoEhER, Routing Metrics Based on Soft-Output Decoding. IEEE, 2007.

[13] K. Homayounfar, Cross-Layer MANET Routing Algorithm Based on Radio Link Stability. IEICE Tech. Rep., 108(447), AN2008-64, pp. 1-6, March 2009.

[14] L. QIN, T. KunZ, Survey on Mobile Ad Hoc Network Routing Protocols and Cross-Layer Design. Carleton University, Systems and Computer Engineering, Technical Report SCE-04-14, August 2004.

[15] L. Mucchi, L. Chisci, L. FABbrini, G. GiovanNETTI, A robust cross-layer metric for routing protocol in mobile wireless ad hoc networks. EURASIP Journal on Advances in Signal Processing 2012.

[16] M. Heni, R. Bouallegue, Power Control in Reactive Routing Protocol for Mobile Ad Hoc Network. International Journal of Wireless \& Mobile Networks (IJWMN), 4(2), April 2012.

[17] Md A. Bin Ngadi, S. Ali, A. H. Abdullah, R. H. KHOKHAR, A taxonomy of cross layer routing metrics for wireless mesh networks. EURASIP Journal on Wireless Communications and Networking 2012.

[18] M. Fatima, R. Gupta, T. K. Bandhopadhyay, Route Discovery by Cross Layer Approach for MANET. International Journal of Computer Applications (0975-8887), 37(7), January 2012. 
[19] M. Drini, T. SAADAWI, Link Lifetime Based Route Selection in Mobile Ad-Hoc Networks. International Journal of Communication Networks and Information Security (IJCNIS), 1(3), December 2009.

[20] S. MEnAKa, M. K. JAYANTHI, Effective Stale Route Management Using Preemptive Routing in DSR. World Applied Sciences Journal, 22(11), 15541560, 2013.

Received: April, 2014

Revised: July, 2014

Accepted: September, 2014

Contact addresses:

S. Menaka

School of Information Technology and Engineering

VIT University

Vellore

Tamil Nadu

India

e-mail: smenaka@vit.ac.in

M. K. Jayanthi

Department of Computer Science and Engineering

King Khalid University

Abha

Saudi Arabia

e-mail: dr.mkjayanthi.kku@gmail.com

PROF. S. MENAKA is currently working as Assistant Professor in the School of Information Technology and Engineering at VIT University. She received the B.E. in computer science engineering from the University of Madras and M.E in systems engineering and operations research from Anna University in 2006 and is pursuing her Ph.D in the area of mobile ad hoc networks. Her research interests include computer networks, network security and cryptography.

DR. M. K. JAYANTHI is working as a Professor at the Department of Computer Science and Engineering, King Khalid University, Saudi Arabia. She received her B.E. in computer science engineering from the University of Madras, M.S. degree from BITs Pilani and Ph.D in computer science from SCSVMV University during 2010. Her research interest includes networking, software engineering, intelligent systems. 